\title{
Uniform, Highly Conductive, and Patterned Transparent Films of a Percolating Silver Nanowire Network on Rigid and Flexible Substrates Using a Dry Transfer Technique
}

\author{
Anuj R. Madaria ${ }^{\S}$, Akshay Kumar ${ }^{\S}$, Fumiaki N. Ishikawa, and Chongwu Zhou ( $\left.\varangle\right)$ \\ Department of Electrical Engineering and Center for Energy Nanoscience and Technology, University of Southern California, Los \\ Angeles, California 90089, USA \\ Received: 17 April 2010 / Revised: 28 May 2010 / Accepted: 5 June 2010 \\ C The Author(s) 2010. This article is published with open access at Springerlink.com
}

\begin{abstract}
Silver nanowire films are promising alternatives to tin-doped indium oxide (ITO) films as transparent conductive electrodes. In this paper, we report the use of vacuum filtration and a polydimethylsiloxane (PDMS)-assisted transfer printing technique to fabricate silver nanowire films on both rigid and flexible substrates, bringing advantages such as the capability of patterned transfer, the best performance among various ITO alternatives ( $10 \Omega /$ sq at $85 \%$ transparency), and good adhesion to the underlying substrate, thus eliminating the previously reported adhesion problem. In addition, our method also allows the preparation of high quality patterned films of silver nanowires with different line widths and shapes in a matter of few minutes, making it a scalable process. Furthermore, use of an anodized aluminum oxide (AAO) membrane in the transfer process allows annealing of nanowire films at moderately high temperature to obtain films with extremely high conductivity and good transparency. Using this transfer technique, we obtained silver nanowire films on a flexible polyethylene terephthalate (PET) substrate with a transparency of $85 \%$, a sheet resistance of $10 \Omega /$ sq, with good mechanical flexibility. Detailed analysis revealed that the Ag nanowire network exhibits two-dimensional percolation behavior with good agreement between experimentally observed and theoretically predicted values of critical volume.
\end{abstract}

\section{KEYWORDS}

Ag nanowire, PDMS transfer, transparent electrode, flexible electronics, percolation network

\section{Introduction}

Materials with a combination of high electrical conductivity and optical transparency are important components of many electronic and optoelectronic devices such as liquid crystal displays, solar cells, and light emitting diodes [1-3]. Doped-metal oxides such as tin-doped indium oxide (ITO) and fluorine-doped tin oxide (FTO) have been leading candidates for

$\bar{\S}$ These authors contributed equally.

Address correspondence to chongwuz@usc.edu these technological applications [4-6]. Although ITO and related materials have witnessed 50 years of extensive research and subsequent applications, recently, there has been a quest to look for materials that can replace ITO as the most important transparent conductive electrode (TCE). This need mainly arises because of the rising cost of ITO (due to low availability of indium sources), its brittleness and hence limited applicability in flexible electronic devices, and the 
high temperature processing step required to fabricate it. Towards this objective, various alternatives have been pursued by different groups, among which thin metal films $[7,8]$ and metal grids $[9,10]$ have shown performance comparable to ITO. However, these approaches require processing and fabrication using high vacuum equipment and the resulting TCEs show limited bending ability. Carbon nanotube (CNT) films [11-15] and, more recently, graphene films [16-19] have attracted significant attention and have been successfully used as the TCE in organic light emitting diodes [20-22] and solar cells [23, 24]. However, their performance in terms of sheet resistance and transparency is still inferior to ITO, creating a need to continue the quest for new materials which can act as cheap and reliable TCEs.

Very recently, two pioneering approaches were reported in which metal nanowire networks were used to fabricate TCEs $[25,26]$ and the resulting metal nanowire TCEs were successfully used in organic solar cells [25]. Both reports represent significant and exciting advancements in the effort to replace ITO; however, the silver nanowires used by Lee et al. [25] had a surfactant coating of polyvinylpyrrolidone (PVP) molecules and required a high temperature $\left(200{ }^{\circ} \mathrm{C}\right)$ annealing step to obtain good conductivity. This limits application of these nanowires on flexible polyethylene terephthalate (PET) as a substrate. The transfer method reported by De et al. [26] enables transfer of silver nanowire films to flexible substrates, but this method suffers from poor adhesion between the nanowire film and PET, and is inherently a chemicalintensive process requiring very careful and exhaustive transfer techniques.

In this paper, we demonstrate a facile polydimethylsiloxane (PDMS)-assisted dry transfer printing technique [15] to fabricate a TCE using Ag nanowires, bringing advantages including easy and fast transfer to both rigid and flexible substrates, good adhesion between the nanowire film and the substrate, and the patterned transfer of various line widths and geometries to obtain pixilated electrodes. Rogers' group has previously extensively studied the stamp-assisted dry printing technique and has demonstrated printing of various metal and semiconductor nanostructures [27-31]. In our case, the use of an anodized aluminum oxide (AAO) membrane allows annealing of nanowires which results in their fusion. In this way, superior quality silver nanowire films were obtained with a sheet resistance $\left(R_{\mathrm{sh}}\right)$ of $10 \Omega / \mathrm{sq}$ at an optical transmittance (T) of $85 \%$. This $R_{\mathrm{sh}}$ value at $85 \%$ transparency is at least an order of magnitude lower than that observed for CNT and graphene films, and is comparable to the values reported for $\mathrm{Ag}$ nanowire electrodes such as $R_{\text {sh }}=13 \Omega /$ sq with $T=85 \%$ [26] and $R_{\text {sh }}=16.1 \Omega /$ sq with $T=86 \%$ [25]. In addition, a detailed study of the conduction behavior of our films using percolation theory [32-34] shows critical exponent value $(\alpha)$ of 1.42 which is indicative of two-dimensional (2-D) percolation behavior, with good agreement between experimentally observed and theoretically predicted values of critical volume.

\section{Experimental}

Silver nanowires used during the fabrication of films were purchased from Seashell Technologies and supplied with a concentration of $12.5 \mathrm{mg} / \mathrm{mL}$ in isopropyl alcohol. The dispersion was further diluted to a concentration of $0.208 \mathrm{mg} / \mathrm{mL}$ in isopropyl alcohol which was subsequently used in all the transfer processes. PDMS stamps were fabricated using a SYLGARD 184 silicone elastomer kit (Dow Corning, Inc.). Patterned PDMS was made by standard optical lithography using an SU-8-50 resist (MicroChem Corp.). The silver nanowire dispersion was vacuum filtered by using an AAO filter ( $47 \mathrm{~mm}, 0.2 \mu \mathrm{m}$, Whatman plc). The nanowire film was transferred onto PDMS by making a conformal contact between the filter and stamp. Glass substrates were functionalized by 3-aminopropyltriethoxysilane (APTES) by dipping in a $1 \mathrm{mmol} / \mathrm{L}$ solution for $20 \mathrm{~min}$ and then blow dried. No functionalization was performed on PET. The receiving substrate was placed on a hotplate at $120^{\circ} \mathrm{C}$, and PDMS was pressed against it for $\sim 1 \mathrm{~min}$. The receiving substrate was removed slowly and the silver nanowire film was obtained.

Optical transmission spectra were obtained using a Varian Cary 50 Conc spectrophotometer, with bare PET used as the reference. Scanning electron microscopy (SEM) micrographs were obtained using a JEOL 7001 field emission scanning electron microscope. Sheet 
resistance was measured using four-probe techniques by depositing silver paint at the corners in a square shape with sides of $\sim 3 \mathrm{~mm}$ and at least 10 locations across the sample, and values reported are the average value obtained across the entire film. An Agilent 4156B Semiconductor Parameter Analyzer was used as a source meter for sheet resistance measurement. The nanowires on the AAO membrane were annealed in air on a hotplate at $200{ }^{\circ} \mathrm{C}$ for various durations. Bending tests were performed by placing the film between two platforms and bending the films by reducing the distance between the platforms. Sheet resistance measurements were performed at various bending angles by placing four probes on the films with silver paint at the corners (see the inset in Fig. 5 for details).

\section{Results and discussion}

Figure 1(a) shows a schematic illustration of the transfer process. Briefly, a measured amount of a silver nanowire suspension (as received, without any intentional surfactant coating) in isopropyl alcohol (IPA) was filtered on a commercially available AAO membrane to obtain a uniform film of nanowires. A PDMS stamp was brought into contact with the AAO membrane and the nanowire film was picked up on the stamp with a yield of $100 \%$. The PDMS stamp bearing the nanowires was pressed against the receiving substrate at a moderate temperature (as described in the Experimental section). The pressure was released after a few minutes and the PDMS stamp was peeled off slowly from the substrate resulting in transfer of the nanowires to the substrate. The entire process takes only a few minutes and the size of the transfer is limited only by the size of the starting AAO membrane. We note that the same PDMS stamp and the AAO membrane can be used multiple times.

We used both glass and PET substrates to transfer
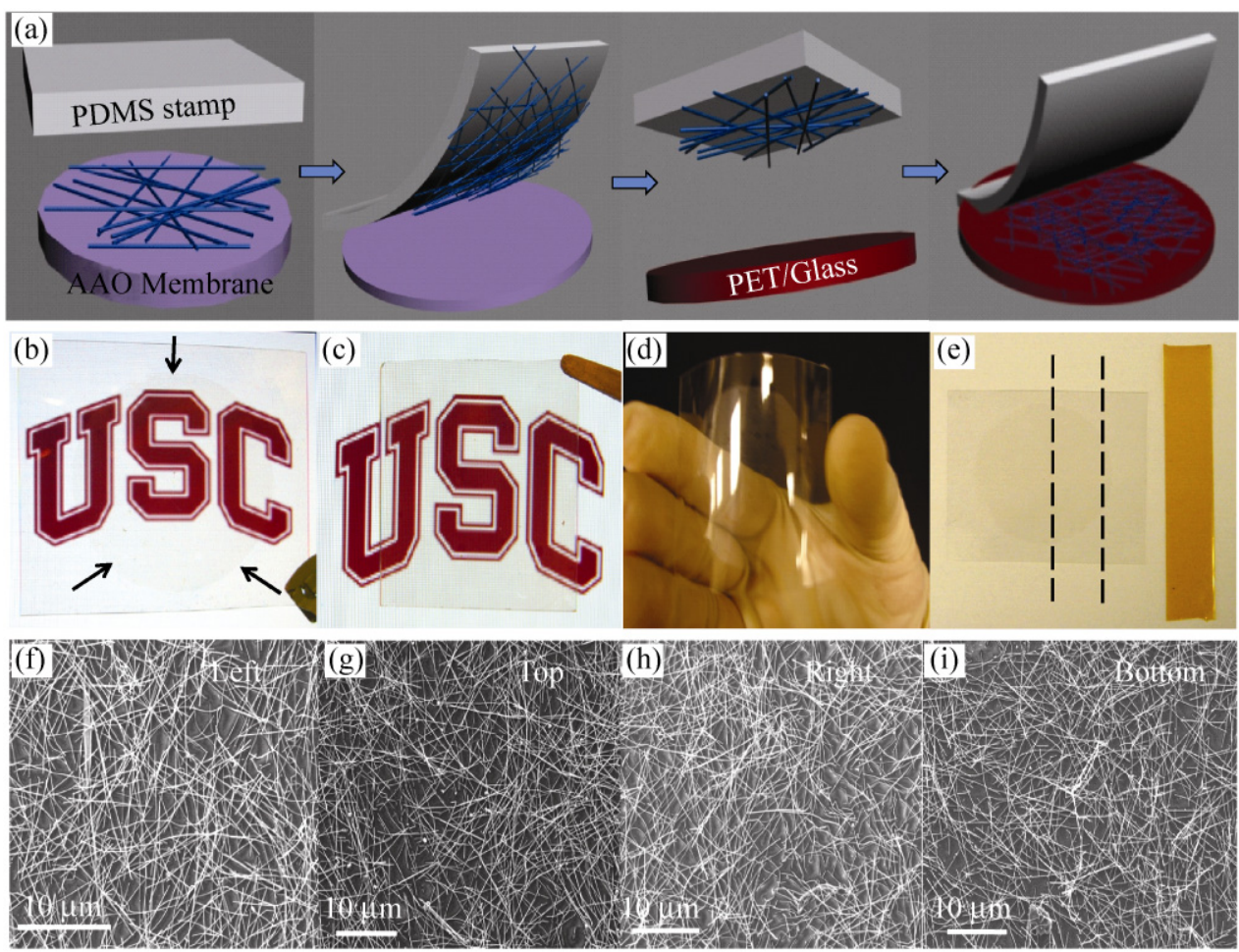

Figure 1 (a) Schematic representation of the transfer process: (i) pressing of the PDMS stamp against the silver nanowire film on the AAO membrane; (ii) peeling off the PDMS stamp; (iii) pressing the PDMS stamp with Ag nanowires on the receiving substrate; (iv) peeling off the PDMS stamp leaving the nanowire film on the receiving substrate. (b) Photograph of a silver nanowire film transferred onto PET. Arrows show the boundary of the nanowire film. (c) Photograph of a nanowire film on a glass substrate. (d) Photograph of a nanowire film on PET showing the flexibility of film. (e) Photograph showing the results of adhesion tests. Nanowires remain adhered to the PET substrate when sticky tape was peeled off from the area shown by dotted lines. SEM images of the (f) left, (g) top, (h) right and (i) bottom regions of the film shown in Fig. 1(b), demonstrating uniformity of the film across the entire area 
large area nanowire films. Figures $1(b)$ and $1(c)$ show optical micrographs of the nanowire films on PET and glass, respectively. The films are highly transparent as the letters in the background can be clearly seen through the film. Figure 1(d) illustrates the flexibility of the transferred film on PET. We note that while the nanowire film could be transferred onto the PET substrate without the aid of any adhesive layer, the film could not be transferred onto the bare glass substrate. This can be explained in terms of the weak adhesion of noble metals such as silver with the highly polar $\mathrm{SiO}_{2}$ surface [35]. On the other hand, the PET surface is characterized by a dominant dispersive component in its surface tension, promoting stronger adhesion to noble metals [35]. In order to achieve good transfer onto the glass substrate, we functionalized the glass surface by depositing a monolayer of APTES molecules, which makes the surface slightly positively charged because of the presence of $\mathrm{NH}_{2}$ groups, facilitating transfer of the nanowires [36]. SEM micrographs of the film transferred onto the PET substrate reveal that transfer process is extremely uniform over the entire area of the film resulting in uniform density of nanowires everywhere on the substrate (Figs. 1(f)-1(i)). We note that our nanowire film adheres strongly to the underlying PET substrate, as opposed to a previous report where the adhesion between nanowires and PET was very weak, and the nanowires could be easily peeled-off from the substrate using sticky tape [26]. We tried to peel off the nanowire film from the PET substrate using sticky tape by firmly attaching it in the region shown by the dotted line in Fig. 1(e), but the nanowires remained on the PET without any visible change to the film, showing their strong adhesion with the substrate. We believe this is a result of strong conformal pressure applied by the PDMS stamp as opposed to the use of a flexible cellulose membrane used in the previous study [26].

The ability to obtain in-plane pixilation is a critical requirement for any display device such as an organic light-emitting diode (OLED). Our dry contact printing method is not only solvent-free, making it an inherently clean process, but also allows patterning of the nanowire film to obtain pixels of nanowires on the substrate. We note that the previously reported cellulose-based transfer method suffers from limitations in its ability to fabricate patterned films over a large area. In our case, patterning is simply achieved by using a patterned PDMS stamp in place of a plane stamp. The transfer process remains the same, giving an unprecedented ability to obtain both patterned and unpatterned uniform nanowire films on any substrate.

The patterned PDMS stamp was fabricated using standard optical lithography. Figure 2(a) shows a schematic illustration of the patterned PDMS stamp and Fig. 2(b) is an optical micrograph of a patterned nanowire film on a PET substrate. The length of the printed squares is $1 \mathrm{~mm}$ with a spacing of $0.5 \mathrm{~mm}$ between them. SEM inspection of the transferred region shows a high density of nanowires on the substrate, suggesting high fidelity of the patterned transfer process (Fig. 2(c)). Several kinds of patterns can be fabricated, with the minimum feature size limited by the resist used in optical lithography to pattern the stamp.

In addition, deposition of the film of silver nanowires on an AAO membrane allows the annealing of the film, resulting in further enhanced electrical conductivity in the films; in contrast, cellulose membranes cannot withstand high temperatures. To study the effects of annealing, we prepared Ag nanowire films of various densities on AAO membranes, and then each sample was divided into two pieces after which one was annealed, and other was not. All the films were then transferred onto PET using a PDMS stamp, followed by thorough characterization. Figure 3(a) shows plots of the sheet resistance vs. transparency of the nanowire films with different densities for samples with and without annealing. The values of the transmittance used for the plot correspond to $\lambda=$ $550 \mathrm{~nm}$ and the full transmittance spectra are shown in the inset of Fig. 3(a) for films with three different densities. Angular dependence of transmittance is shown in Fig. S-1 in the Electronic Supplementary Material (ESM). The transmittance changed by $\sim 10 \%$ when the incident angle of the light was changed from $0^{\circ}$ to $60^{\circ}$ with respect to the substrate normal. As expected, samples with higher transparency typically showed higher sheet resistance. Importantly, there was a significant decrease in the sheet resistance for films annealed at $200{ }^{\circ} \mathrm{C}$ for 20 minutes. For example, a sample with a transparency of $85 \%$ (at a wavelength 

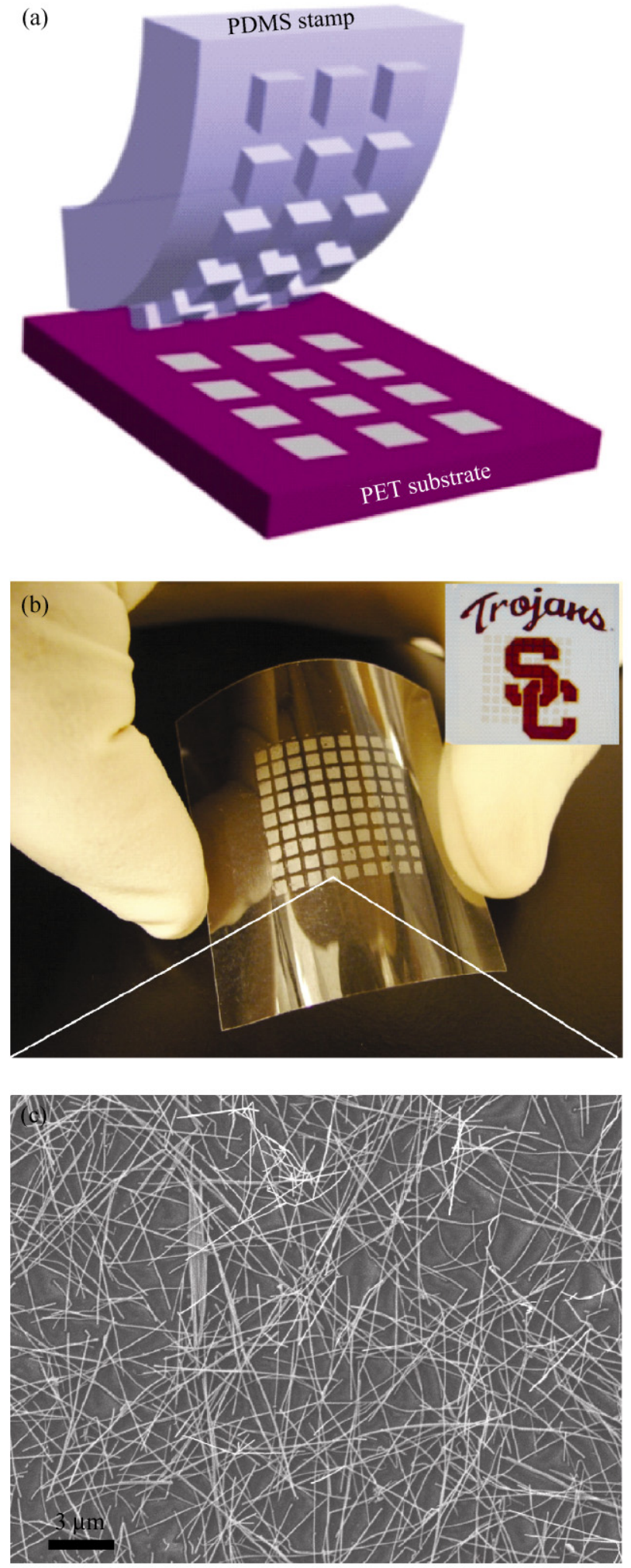

Figure 2 Patterned transfer of silver nanowire film. (a) Schematic diagram of the patterned PDMS stamp in contact with the nanowire film. (b) Photograph of patterned nanowire film transferred on the PET substrate. The size of each pixel is $1 \mathrm{~mm} \times 1 \mathrm{~mm}$. (c) SEM image showing the nanowire network in one pixel of $550 \mathrm{~nm}$ ) had a sheet resistance of $30 \Omega /$ sq without annealing, while the corresponding annealed sample showed a sheet resistance of $10 \Omega /$ sq. The sheet resistance of $10 \Omega / \mathrm{sq}$ at $85 \%$ transparency for our nanowire film is comparable to both drop-coated films $(16.1 \Omega / \mathrm{sq}$ at $T=86 \%$ ) [25] and cellulose membrane assisted films (13 $\Omega / \mathrm{sq}$ at $T=85 \%)$ [26]. We note that different preparation techniques are likely to be used for different applications, and further development of all three preparation methods may lead to even better performance. We also note that all our reported sheet resistance values are the average of many data points measured for different parts of the sample, giving a more accurate representation of the conductivity of the sample. Statistical data for the variation of sheet resistance values for a film with transmittance of $85 \%$ are shown in Fig. S-2 in the ESM. We attribute the increase in conductivity after annealing to the better contact and fusion between the nanowires. Similar results were obtained by Lee et al. [25]; however in their case, the primary objective for carrying out an annealing step was to remove the PVP-capping agents surrounding the nanowires during the synthesis. SEM images of the nanowires annealed on an AAO membrane clearly show melting and fusion of nanowires leading to superior contact between them (Fig. 3(b)). This further reduces the contact resistance between nanowires, resulting in highly conductive nanowire films.

In order to optimize the annealing temperature and annealing time, we prepared samples with different densities of nanowires, corresponding to initial volumes of $\mathrm{Ag}$ nanowire solution of $0.3 \mathrm{~mL}, 0.75 \mathrm{~mL}$, $3 \mathrm{~mL}$, and $8 \mathrm{~mL}$, as shown in Fig. 3(d). It is evident from the figure that the nanowires form a percolated network which becomes denser with increasing dispersion volume. We then carried out a time-dependent annealing study, with representative results shown in Fig. 3(c) for samples using $0.3 \mathrm{~mL}, 0.75 \mathrm{~mL}$, and $3 \mathrm{~mL}$ of starting solution. A general trend of initial decrease and subsequent increase in sheet resistance with increasing annealing time can be seen for all the samples in Fig. 3(c). With a further increase in annealing time, the sheet resistance starts to increase because of droplet formation, consistent with the observation of 
(a)

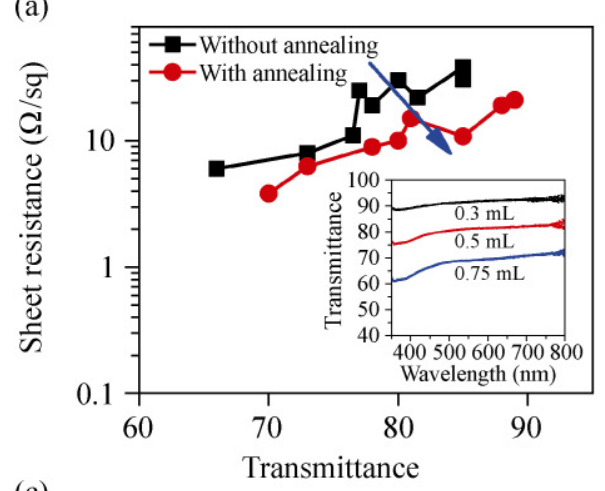

(c)

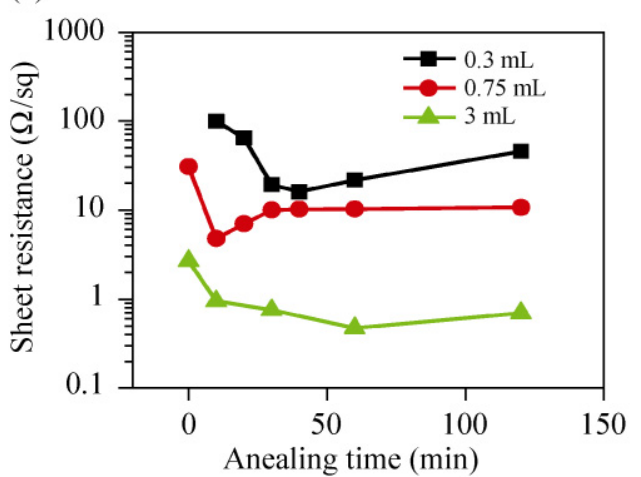

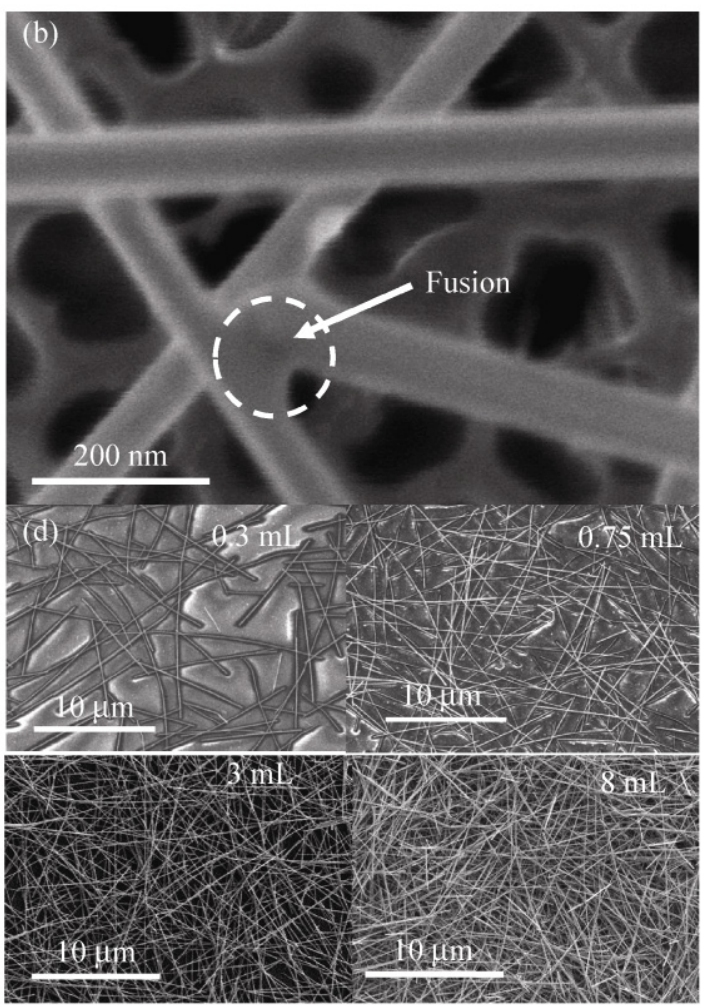

Figure 3 (a) Plot of sheet resistance versus transmittance at $\lambda=550 \mathrm{~nm}$ of silver nanowire films. Black dots represent resistance values for films without annealing and red dots represent resistance values after annealing the film samples at $200{ }^{\circ} \mathrm{C}$ for 20 min in air. Inset shows the transmittance spectra of nanowire films with different thicknesses. (b) SEM image of an annealed nanowire sample showing melting of nanowires at the ends and subsequent joining with neighboring nanowires. (c) Variation of sheet resistance with the annealing time for nanowires films of different densities. Sheet resistance decreased initially on annealing and subsequently increased with further annealing (d) SEM images of nanowire films prepared using various amounts of nanowire solution. Nanowire density increases with increased volume of solution

Lee et al. [25]. When the film is heated to $300{ }^{\circ} \mathrm{C}$, the nanowires melt completely forming small droplets and losing all conductivity shown in Fig. S-3 in the ESM. This systematic study therefore gives an optimized annealing temperature of $200{ }^{\circ} \mathrm{C}$ and an optimized time of $20 \mathrm{~min}$ in order to obtain highly conductive films without disrupting the integrity of the nanowire network. A decrease in the sheet resistance of all the films by a factor of three was observed after annealing. A two-probe measurement of the nanowire film showed linear current-voltage characteristics, demonstrating the ohmic behavior of the nanowire film shown in Fig. S-4 in the ESM. Nanowire films showed no appreciable change in sheet resistance after exposing the films to ambient conditions for 90 days.

Further analysis of Ag nanowire films was carried out by studying the percolation behavior of the network. For a given volume of the nanowire solution $(V)$, with a concentration of $C(0.208 \mathrm{mg} / \mathrm{mL})$, the number of nanowires can be calculated by the equation

$$
\text { No. of nanowires }=\frac{4 C V}{D_{\mathrm{Ag}} \pi d^{2} L}
$$

where $D_{\mathrm{Ag}}$ is the density of bulk silver $(10.5 \mathrm{~g} / \mathrm{mL}), d$ is the average diameter $(75 \mathrm{~nm})$, and $L$ is the average length $(12.5 \mu \mathrm{m})$ of the Ag nanowire provided by the supplier. After vacuum filtration, these nanowires were evenly distributed on the AAO membrane of $47 \mathrm{~mm}$ diameter $(D)$. Hence, the density of nanowires, $N$ can be calculated by dividing the number of nanowires by the area of the AAO membrane, and is given by

$$
N=\frac{4 C V}{D_{\mathrm{Ag}} \pi d^{2} L} / \pi D^{2} / 4
$$

Substituting the corresponding values in the above

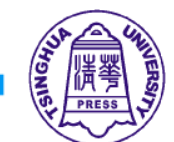


equation gives $N=0.20697 \mathrm{~V}_{\mu \mathrm{m}}^{-2}$ for a given volume $(V)$ of the nanowire solution used. According to the standard percolation theory, the density dependence of conductivity is given by

$$
\sigma \propto\left(N-N_{\mathrm{c}}\right)^{\alpha}
$$

where $\sigma$ is the conductivity in three dimensions, or sheet conductance in two dimensions, $N_{\mathrm{c}}$ is the critical nanowire density required for the onset of conduction in a random network, and $\alpha$ is a critical exponent which depends on the dimensionality of the space involved. The theoretical values of $\alpha$ are 1.33 for a 2-D percolation network and 1.94 for a 3-D percolation network [37]. For a 2-D network, the sheet resistance $\left(R_{\mathrm{sh}}\right)$ is given by $R_{\mathrm{sh}}=1 /$ sheet conductance, and the relation between $R_{\text {sh }}$ and volume (V) of the nanowire solution can be obtained using Eq. (3) as follows:

$$
R_{\mathrm{sh}} \propto\left(V-V_{\mathrm{c}}\right)^{-\alpha}
$$

Figure 4 shows the variation of sheet resistance with the volume of nanowire solution used and the solid line represent the fitted curve obtained using Eq. (4). In order to obtain the fitting curve, we took the value of $V_{c}$ as $0.20 \mathrm{~mL}$ as this was the minimum volume required to achieve a conducting film (no conductive film was obtained by using $0.15 \mathrm{~mL}$ of nanowire solution). Theoretically, for a model involving random distribution of nanowires [32], $N_{\mathrm{c}}$ for nanowires with length $l$ is given by

$$
l \sqrt{\pi N_{\mathrm{c}}}=4.236
$$

Substituting the length of Ag nanowires $(12.5 \mu \mathrm{m})$ in the above equation, we can calculate the critical density for the network to be $0.03657 \mu \mathrm{m}^{-2}$. Substituting this critical density in Eq. (2), the resulting theoretical critical volume $V_{\mathrm{c}}$ is $0.18 \mathrm{~mL}$, which is very close to the experimentally observed critical volume of $0.20 \mathrm{~mL}$. We note that the value of the critical volume obtained experimentally is a little higher than the theoretical value, which might be due to the formation of multilayer stacking of nanowires in some regions rather than an ideal monolayer.

The best fit of Eq. (4) in Fig. 4 is obtained by using

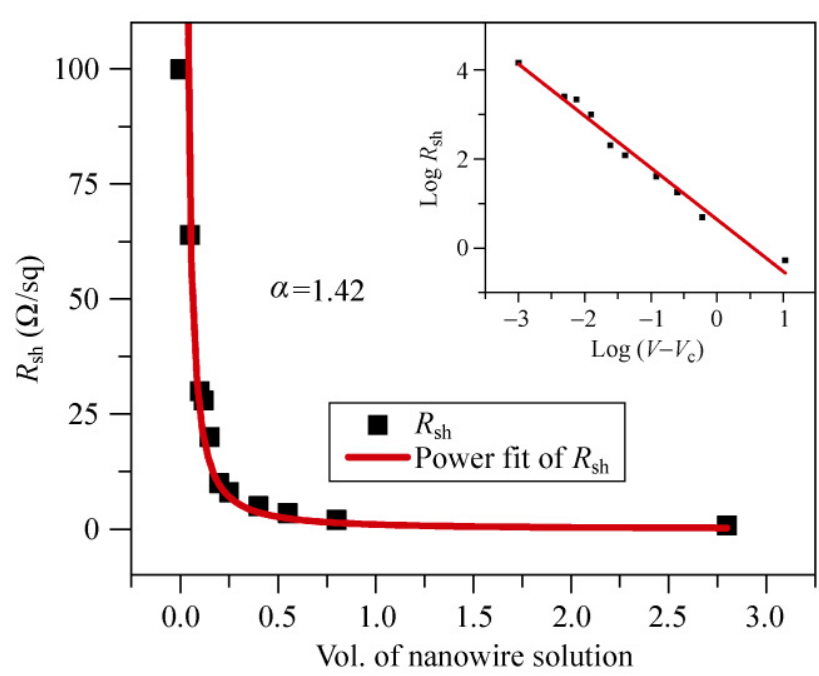

Figure 4 Plot of sheet resistance versus volume of Ag nanowire solution. The onset of conduction across the sample occurs for $V_{\mathrm{c}}=0.2 \mathrm{~mL}$. The power fit of the data indicated value of critical exponent $\alpha=1.42$. The inset shows the logarithmic plot of the data with a linear fit

$\alpha=1.42$, which is quite close to the value predicted for the 2-D theoretical model. The discrepancy between experimental and theoretical values may indicate that the nanowire film is not a perfect 2-D film, and actually has an intermediate character between 2-D and 3-D; as a result, the experimental value is between that for 2-D $(\alpha=1.33)$ and 3-D $(\alpha=1.94)$. The inset shows the logarithmic fit of the above equation, resulting in a linear plot with good agreement between the experimental and predicted values.

Another major disadvantage of ITO films as TCEs lies in their brittleness, which restricts their application in flexible electronics [38]. Flexible electronic and optoelectronic devices have attracted a lot of interest in past few years with many groups reporting flexible OLEDs [39], flexible photovoltaic devices [40], flexible supercapacitors [41], and flexible logic inverters [42], among other applications. Silver nanowire films offer the required flexibility and are a potential replacement for ITO in flexible TCEs. An added attraction of the silver nanowire film is that this advantage of flexibility does not come at the cost of performance in terms of sheet resistance and transparency. As mentioned earlier, a value of $R_{\mathrm{sh}}$ of $10 \Omega / \mathrm{sq}$ at $T=85 \%$ for a nanowire film is very comparable to the performance of ITO. In 
order to test the durability of nanowire films under stress, we measured their sheet resistance at different bending angles. The results are shown in Fig. 5. The inset shows the setup for the measurement of change in sheet resistance with bending angle, where the bending angle is the angle between the tangents drawn from the bent substrate. The sheet resistance varied from $11 \Omega /$ sq to only $17 \Omega /$ sq when subjected to a bending angle of up to $160^{\circ}$. Using an equivalent thickness of $100 \mathrm{~nm}$ for a film with a transmittance of $80 \%$ [26], we estimate that the average normal strain value [43] varies from 0.012 to 0.007 for bending angles from $10^{\circ}$ to $160^{\circ}$. No failure was observed up to a bending angle of $160^{\circ}$. The overall integrity and conductivity of nanowire films remained intact when the stress was released. SEM inspection revealed that there was no appreciable change in nanowire morphology or the network (images not shown). In comparison, the conductivity of an ITO film on PET substrate was reduced by three orders of magnitudes when it was bent to an angle of only $60^{\circ}$, demonstrating its limitations for applications in flexible devices [44].

We have to note that complete transfer for samples with higher densities (corresponding to $T<60 \%$ ) could not be achieved consistently, as some of the nanowires remained adhered to the PDMS membrane. Also, the

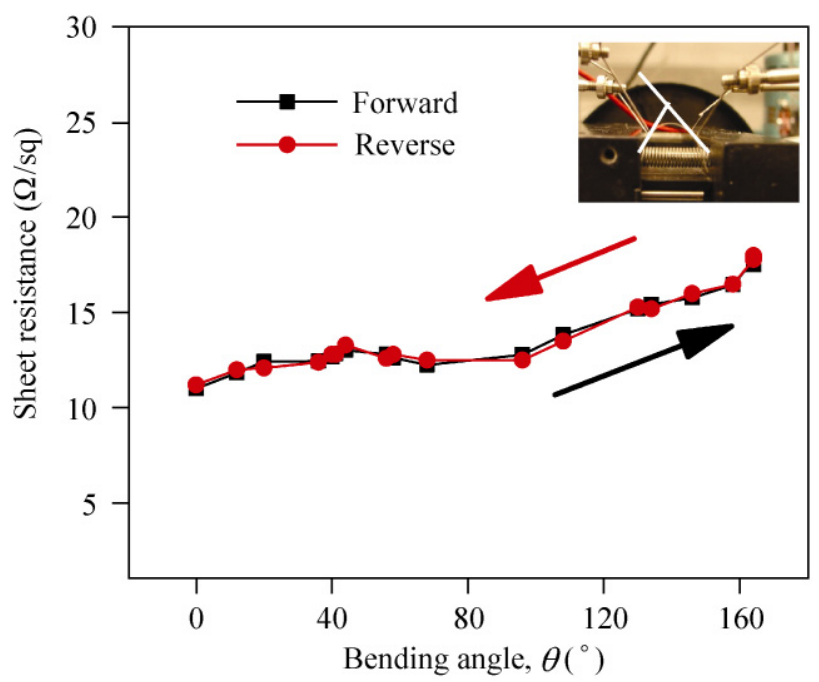

Figure 5 Plot of sheet resistance versus bending angle. The nanowire film remains conducting under severe bending. Inset shows the photograph of the measurement setup and definition of the bending angle adhesion of nanowires to the substrate decreased with the increasing density of nanowires on the substrate. We believe this is due to lack of interaction between nanowires which are further away from the substrate and the substrate, which results in poor adhesion of the nanowires. Although this is a problem, it occurs only in films with lower transmittance which do not find significant applications in optoelectronic devices.

\section{Conclusions}

In summary, we have demonstrated a simple, rapid, chemical-free, dry transfer printing technique for fabricating high quality silver nanowire films on both glass and PET substrates. The PDMS-assisted transfer affords extremely uniform films with strong adhesion to a PET substrate over a large area. Use of a patterned PDMS stamp gives the ability to obtain patterned nanowire films with high fidelity. Films thus obtained have extremely low sheet resistance of $10 \Omega / \mathrm{sq}$ at a transparency of $85 \%$ which is comparable to ITO films. The nanowire films form a 2-D percolation network and retain their good conductivity even under bending, and recover their original low resistance once the stress is released.

\section{Acknowledgements}

This material is based upon work supported as part of the Center for Energy Nanoscience and Technology (CENT), an Energy Frontier Research Center (EFRC) funded by the U.S. Department of Energy, Office of Science and Office of Basic Energy Sciences under Award No. DE-SC0001013.

Electronic Supplementary Material: Supplementary material is available in the online version of this article at http://dx.doi.org/10.1007/s12274-010-0017-5 and is accessible free of charge.

Open Access: This article is distributed under the terms of the Creative Commons Attribution Noncommercial License which permits any noncommercial use, distribution, and reproduction in any medium, provided the original author(s) and source are credited. 


\section{References}

[1] Haacke, G. Transparent conducting coatings. Ann. Rev. Mater. Sci. 1977, 7, 73-93.

[2] Granqvist, C. G. Transparent conductors as solar energy materials: A panoramic review. Sol. Energy Mater. Sol. Cells 2007, 91, 1529-1598.

[3] Thomas, G. Invisible circuits. Nature 1997, 389, 907-908.

[4] Minami, T. Transparent conducting oxide semiconductors for transparent electrodes. Semicond. Sci. Technol. 2005, 20, S35-S44.

[5] Chopra, K. L.; Major, S.; Pandya, D. K. Transparent conductors-A status review. Thin Solid Films 1983, 102, $1-46$.

[6] Hartnagel, H. L.; Dawar, A. L.; Jain, A. K.; Jagadish, C. Semiconducting Transparent Thin Films; Institute of Physics Publishing: Philadelphia, 1995.

[7] Meiss, J.; Riede, M. K.; Leo, K. Towards efficient tindoped indium oxide (ITO)-free inverted organic solar cells using metal cathodes. Appl. Phys. Lett. 2009, 94, 013303.

[8] O'Connor, B.; Haughn, C.; An, K. H.; Pipe, K. P.; Shtein, M. Transparent and conductive electrodes based on unpatterned, thin metal films. Appl. Phys. Lett. 2008, 93, 223304.

[9] Kang, M. G.; Kim, M. S.; Kim, J. S.; Guo, L. J. Organic solar cells using nanoimprinted transparent metal electrodes. $A d v$. Mater. 2008, 20, 4408-4413.

[10] Tvingstedt, K.; Inganas, O. Electrode grids for ITO-free organic photovoltaic devices. Adv. Mater. 2007, 19, 2893-2897.

[11] Hu, L.; Hecht, D. S.; Gruner, G. Percolation in transparent and conducting carbon nanotube networks. Nano Lett. 2004, 4, 2513-2517.

[12] Zhang, M.; Fang, S.; Zakhidov, A. A.; Lee, S. B.; Aliev, A. E.; Williams, C. D.; Atkinson, K. R.; Baughman, R. H. Strong, transparent, multifunctional, carbon nanotube sheets. Science 2005, 309, 1215-1219.

[13] Geng, H. -Z.; Lee, D. S.; Kim, K. K.; Han, G. H.; Park, H. K.; Lee, Y. H. Absorption spectroscopy of surfactant-dispersed carbon nanotube film: Modulation of electronic structure. Chem. Phys. Lett. 2008, 455, 275-278.

[14] Doherty, E. M.; De, S.; Lyons, P. E.; Shmeliov, A.; Nirmalraj, P. N.; Scardaci, V.; Joimel, J.; Blau, W. J.; Boland, J. J.; Coleman, J. N. The spatial uniformity and electromechanical stability of transparent, conductive films of single walled nanotubes. Carbon 2009, 47, 2466-2473.

[15] Zhou, Y.; Hu, L.; Grüner, G. A method of printing carbon nanotube thin films. Appl. Phys. Lett. 2006, 88, 123109.

[16] Geim, A. K.; Novoselov, K. S. The rise of graphene. Nat. Mater. 2007, 6, 183-191.

[17] Novoselov, K. S.; Jiang, Z.; Zhang, Y.; Morozov, S. V.;
Stormer, H. L.; Zeitler, U.; Mann, J. C.; Boebinger, G. S.; Kim, P.; Geim, A. K. Room-temperature quantum Hall effect in graphene. Science 2007, 315, 1379-1383.

[18] Eda, G.; Fanchini, G.; Chhowalla, M. Large-area ultrathin films of reduced graphene oxide as a transparent and flexible electronic material. Nat. Nanotechnol. 2008, 3, 270-274.

[19] Tung, V. C.; Allen, M. J.; Yang, Y.; Kaner, R. B. Highthroughput solution processing of large-scale graphene. Nat. Nanotechnol. 2009, 4, 25-29.

[20] Zhang, D.; Ryu, K.; Liu, X.; Polikarpov, E.; Ly, J.; Thompson, M. E.; Zhou, C. Transparent, conductive, and flexible carbon nanotube films and their application in organic light-emitting diodes. Nano Lett. 2006, 6, 1880-1886.

[21] Li, J.; Hu, L.; Wang, L.; Zhou, Y.; Gruner, G.; Marks, T. J. Organic light-emitting diodes having carbon nanotube anodes. Nano Lett. 2006, 6, 2472-2477.

[22] Wu, J.; Agarwal, M.; Becerril, H. A.; Bao, Z.; Liu, Z.; Chen, Y.; Peumans, P. Organic light-emitting diodes on solutionprocessed graphene transparent electrodes. ACS Nano 2010, $4,43-48$.

[23] Rowell, M. W.; Topinka, M. A.; McGehee, M. D.; Prall, H. -J.; Dennler, G.; Sariciftci, N. S.; Hu, L.; Grüner, G. Organic solar cells with carbon nanotube network electrodes. Appl. Phys. Lett. 2006, 88, 233506.

[24] Wu, J.; Becerril, H. A.; Bao, Z.; Liu, Z.; Chen, Y.; Peumans, P. Organic solar cells with solution-processed graphene transparent electrodes. Appl. Phys. Lett. 2008, 92, 263302.

[25] Lee, J. -Y.; Connor, S. T.; Cui, Y.; Peumans, P. Solutionprocessed metal nanowire mesh transparent electrodes. Nano Lett. 2008, 8, 689-692.

[26] De, S.; Higgins, T. M.; Lyons, P. E.; Doherty E. M.; Nirmalraj, P. N.; Blau, W. J.; Boland, J. J.; Coleman, J. N. Silver nanowire networks as flexible, transparent, conducting films: Extremely high DC to optical conductivity ratios. ACS Nano 2009, 3, 1767-1774.

[27] Zaumseil, J.; Someya, T.; Baldwin, K.; Bao, Z.; Loo, Y. -L.; Rogers, J. A. Nanoscale organic transistors that use source/ drain electrodes supported by high resolution rubber stamps. Appl. Phys. Lett. 2003, 82, 793-795.

[28] Choi, K. M.; Rogers, J. A. A photocurable poly(dimethylsiloxane) chemistry designed for soft lithographic molding and printing in the nanometer regime. J. Am. Chem. Soc. 2003, 125, $4060-4061$

[29] Lee, T. -W.; Zaumseil, J.; Bao, Z.; Hsu, J. W. P.; Rogers, J. A. Organic light-emitting diodes formed by soft contact lamination. Proc. Nat. Acad. Sci. USA 2004, 101, 429-433.

[30] Sun, Y.; Rogers, J. A. Fabricating semiconductor nano/ microwires and transfer printing ordered arrays of them onto plastic substrates. Nano Lett. 2004, 4, 1953-1959. 
[31] Meitl, M. A.; Zhu, Z. -T.; Kumar, V.; Lee, K. J.; Feng, X.; Huang, Y. Y.; Adesida, I.; Nuzzo, R. G.; Rogers, J. A. Transfer printing by kinetic control of adhesion to an elastomeric stamp. Nat. Mater. 2006, 5, 33-38.

[32] Pike, G. E.; Seager, C. Percolation and conductivity: A computer study. I. Phys. Rev. B 1974, 10, 1421-1434.

[33] Yi, Y.; Sastry, A. Analytical approximation of the twodimensional percolation threshold for fields of overlapping ellipses. Phys. Rev. E 2002, 66, 066130.

[34] Kirkpatrick, S. Percolation and conduction. Rev. Mod. Phys. 1973, 45, 574-588.

[35] Hines, D. R.; Mezhenny, S.; Breban, M.; Williams, E. D.; Ballarotto, V. W.; Esen, G.; Southard, A.; Fugrer, M. S. Nanotransfer printing of organic and carbon nanotube thinfilm transistors on plastic substrates. Appl. Phys. Lett. 2005, 86, 163101.

[36] Freeman, R. G.; Graber, K. C.; Allison, K. J.; Bright, R. M.; Davis, J. A.; Guthrie, A. P.; Hommer, M. B.; Jackson, M. A.; Smith, P. C.; Walter, D. G.; Natan, M. J. Self-assembled metal colloid monolayers: An approach to SERS substrates. Science 1995, 267, 1629-1632.

[37] Stauffer, G. Introduction to Percolation Theory; Taylor \& Francis: London, 1985.

[38] Crawford, G. P. Flexible Flat Panel Displays; John Wiley \& Sons: England, 2005.
[39] Li, J.; Liu, J.; Wang, L.; Marks, T. J.; Hu, L.; Grüner, G. Indium tin oxide modified transparent nanotube thin films as effective anodes for flexible organic light-emitting diodes. Appl. Phys. Lett. 2008, 93, 083306.

[40] Pagliaro, M.; Ciriminna, R.; Palmisano, G. Flexible solar cells. ChemSusChem 2008, 1, 880-891.

[41] Chen, P. -C.; Shen, G.; Sukcharoenchoke, S.; Zhou, C. Flexible and transparent supercapacitor based on $\operatorname{In}_{2} \mathrm{O}_{3}$ nanowire/carbon nanotube heterogeneous films. Appl. Phys. Lett. 2009, 94, 043113.

[42] Ishikawa, F. N.; Chang, H. -K.; Ryu, K.; Chen, P. -C.; Badmaev, A.; De Arco, L. G.; Shen, G.; Zhou, C. Transparent electronics based on transfer printed aligned carbon nanotubes on rigid and flexible substrates. ACS Nano 2009, 3, 73-79.

[43] Dikin, D. A.; Stankovich, S.; Zimney, E. J.; Piner, R. D.; Dommett, H. B.; Evmenenko, G.; Nguyen, S. T.; Ruoff, R. $\mathrm{S}$. Preparation and characterization of graphene oxide paper. Nature 2006, 448, 457-460.

[44] Tung, V. C.; Chen, L.; Allen, M. J.; Wassei, J. K.; Nelson, K.; Kaner, R. B.; Yang, Y. Low-temperature solution processing of graphene-carbon nanotube hybrid materials for highperformance transparent conductors. Nano Lett. 2009, 9, 1949-1955. 